\title{
Regenerating MR Tagged Images Using Harmonic Phase (HARP) Methods
}

\author{
Nael F. Osman* and Jerry L. Prince, Fellow, IEEE
}

\begin{abstract}
Magnetic resonance tagging has proven useful in the visualization and quantification of cardiac motion. Traditionally, tags are designed to have crisp geometric profiles in order to enhance both visualization and detection of tags. Recent image acquisition and analysis methods, however, have been designed to exploit sinusoidal tag profiles. This paper presents a method based on harmonic phase (HARP) concepts to synthesize tag lines that have both crisp profiles and alternative orientations from the original sinusoidal patterns. Results are demonstrated on images acquired with SPAMM, CSPAMM, and fast-HARP pulse sequences.
\end{abstract}

Index Terms-Harmonic phase (HARP), magnetic resonance (MR) tagging, motion tracking, regional function.

\section{INTRODUCTION}

$\mathbf{M}$ AGNETIC RESONANCE (MR) tagging has proven to be a useful technique for visualization and quantification of tissue motion and strain [1]-[3]. Tags form regular patterns, typically an array or grid of straight lines, temporarily imposed within the tissue such that they deform with the motion of the tissue. Tissue motion is typically measured by detecting the positions of the tag lines or intersections and tracking their movement over time [4]-[9]. Motion measurement accuracy depends in part on the ability to localize a tag line, which in turn depends on its profile, i.e., the image intensities orthogonal to the tag direction. Thin tag lines, for example, have a narrow intensity profile, which provides good localization provided there is adequate spatial sampling in the acquired image. Such tag lines, however, have very large $k$-space coverage, requiring long acquisition times to image properly. The reason is that sharper tag profiles require higher resolution images, which require higher image resolution, which corresponds to acquiring larger regions in $k$ space.

Several tagged imaging and analysis techniques such as CSPAMM [10]-[12], and HARP [6], [7], [12], [13] have concentrated on sinusoidal tag patterns. In other sequences, such as higher order SPAMM [2] and DANTE [14], the tags were designed to have very sharp profiles, but only a fraction of $k$ space was imaged [15]. In all these cases, the resulting tag

\footnotetext{
Manuscript received March 11, 2003; revised November 11, 2003. This work was supported by the Whitaker Foundation under a Biomedical Engineering Research grant and by the National Heart, Lung, and Blood Institute under Grant R01HL4705. N. F. Osman and J. L. Prince are founders of and own stock in Diagnosoft, Inc., a company that seeks to license the HARP technology. The terms of this arrangement are being managed by the Johns Hopkins University in accordance with its conflict of interest policies. Asterisk indicates corresponding author:

*N. F. Osman is with the Department of Radiology, Johns Hopkins University, Baltimore, MD 21287 USA (e-mail: nael@jhu.edu).

J. L. Prince is with the Electrical and Computer Engineering Department, Johns Hopkins University, Baltimore, MD 21218 USA (e-mail: prince@ jhu.edu).

Digital Object Identifier 10.1109/TBME.2004.827932
}

patterns lack the detail that is necessary for visualization and detection (although they still may have excellent quantification properties, cf., [6], [7], and [13]). In this paper, we propose a technique to synthesize more desirable tag patterns, with both new profiles and tag orientations, from these conventional SPAMM, CSPAMM, and fast-HARP [16] images. This improves the qualitative interpretation of motion by observers and maybe the ability to detect tag lines while simultaneously permitting optimization of the imaging protocol (e.g., [17]).

\section{THEORY}

Our method is based on the principles of harmonic phase (HARP) magnetic resonance imaging (MRI) [6], [7], [13]. Because tag patterns are typically periodic, they can be expanded in a Fourier series. Multiplication of such a tag pattern with the object creates an amplitude modulation that causes $k$ space to have periodically distributed "lumps" of energy called harmonic peaks. The spacing between the peaks is inversely proportional to tag spacing, while the number of the harmonic peaks and their energy ratios affect the width and profile of the resulting tag lines. In general, a smaller number of peaks produce images with wider tag lines than those with a larger number of peaks. For example, 1-1 SPAMM uses only two RF pulses, resulting in three harmonic peaks (including the "dc" peak at the origin of $k$ space) and the tag profile is a simple sinusoid. In this case, we can write the intensity of a tagged MR image, just after tagging, as

$$
I(x, y)=I_{0}(x, y)\left(\frac{1}{2}+\frac{1}{2} \cos \left(\omega_{x} x\right)\right)
$$

where $I_{0}(x, y)$ is the image intensity at some location-that would have been obtained - before tagging and $\omega_{x}$ is the tagging frequency in the $x$ direction (if the tag pattern is vertical line, for example). Based on (1), we can expand the cosine on the right hand into two complex harmonics and obtain three terms on the right-hand side, which will correspond to three harmonic peaks. To obtain crisp thin tag lines, more RF pulses are used, and the resulting images have a larger number of peaks, in general.

The basic concept behind HARP is that each (non-dc) harmonic peak has complete information about a particular one-dimensional (1-D) component of the tag's deformation, which relates to tissue motion. Because of this, HARP uses two harmonic peaks, either directly acquired or extracted using bandpass filters, to estimate the two-dimensional (2-D) motion in a plane. As an example, Fig. 1(a) shows a tagged MR image of the short axis slice of a dog heart. The magnitude of the Fourier transform of this image [Fig. 1(b)] contains periodically replicated 

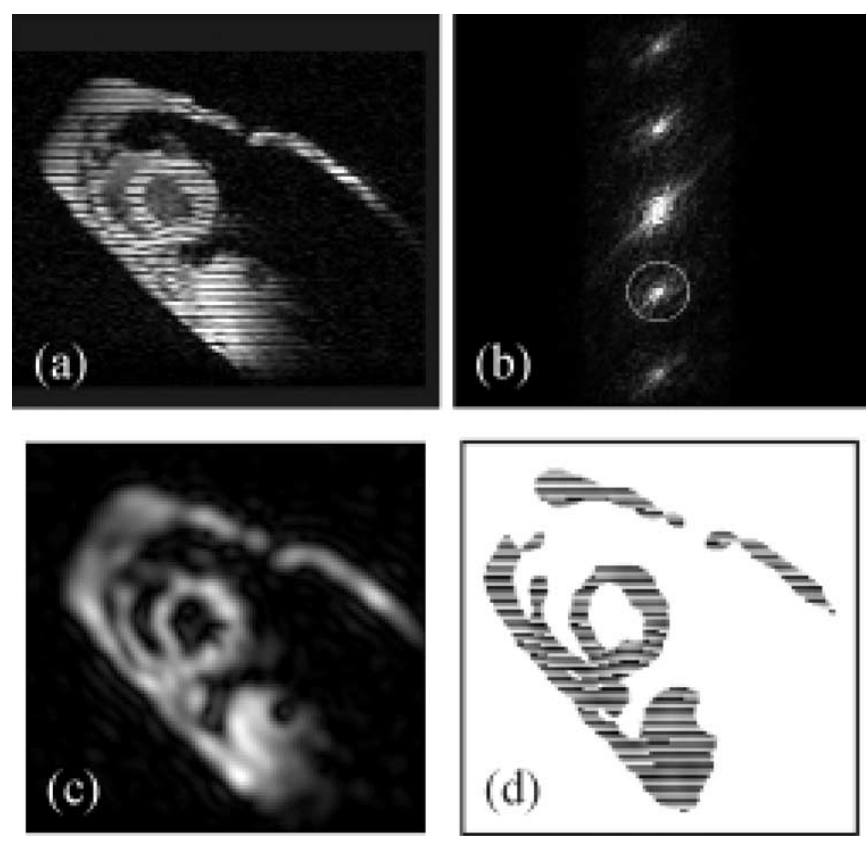

Fig. 1. Panel (a) shows a tagged MR image of a heart with slight contraction Corresponding Fourier space, shown in (b), consists of multiple harmonic peaks because of the tagging. Image corresponding to the peak marked with a circle is a complex image whose magnitude and phase are shown in (c) and (d), respectively.

harmonic peaks corresponding to a set of scaling and shifts of the underlying Fourier transform due to amplitude modulation by the periodic tag pattern. By filtering out the harmonic peak shown in Fig. 1(b) and taking its inverse Fourier transform, a complex harmonic image is produced. The magnitude and phase images corresponding to this harmonic image are shown in Fig. 1(c) and (d), respectively. The phase image, which we call a HARP image, has been masked by a thresholded magnitude image so that only the phase of tissue is shown. The crisp features in the HARP image, which are caused by phase wrapping, bend the same way as that of the tag lines in the original tagged image. This shows the relation between the HARP image and the deformation of the tag pattern due to motion.

Based on the harmonic expansion of an MR tagged image, we can write any image with a periodic tag pattern (lines or grid) as a summation

$$
\mathbf{I}=\sum_{\ell=-L}^{+L} \mathbf{I}_{\ell}
$$

where each term is a harmonic image that corresponds to a spectral peak in the Fourier space of the image. The terms in the summation correspond to the harmonic peaks, which are expected to be symmetrical around the origin of the $k$ space. Except for the dc peak $\mathbf{I}_{0}$ at the origin, the rest of peaks can be grouped into pairs symmetrically around the origin of the Fourier space ( $k$ space); hence, they can have positive and negative indexes. These harmonic images have complex values, in general, and any of them can be written as

$$
\mathbf{I}_{\ell}=\mathbf{D}_{\ell} \exp \left(j \Phi_{\ell}\right)
$$

where the components on the right-hand side are real and can be written in relation to each other by

$$
\begin{aligned}
\mathbf{D}_{\ell} & =c_{\ell} \mathbf{D}_{1} \\
\boldsymbol{\Phi}_{\ell} & =\ell \boldsymbol{\Phi}_{1}+\theta_{\ell} .
\end{aligned}
$$

The image $\mathbf{D}_{\ell}$ is the harmonic magnitude image, affected by falling levels of intensity with time due to tag fading, and $\boldsymbol{\Phi}_{\ell}$ is the harmonic phase image [6], [7], [13]. Notice that the selection of the first harmonic as the reference for the other harmonics in (4) is arbitrary and any other harmonic image, except that of the dc, can replace the first harmonic image. The real coefficients $\left\{c_{\ell}\right\}$ and $\left\{\theta_{\ell}\right\}$, for $\ell=0, \pm 1, \ldots, \pm L$, are responsible for the detailed appearance of the tag profile. The tag profile coefficients depend on two factors. First, they depend on the tagging pulse sequence, specifically the number of the rf pulses that determines the number and values of these coefficients. For example, two rf pulses are used in 1-1 SPAMM to generate a sinusoidal tag profile with only three coefficients (including the dc). In SPAMM tags, the distribution of the coefficients depends on the tipping angles of the RF pulses. The other factor that determines the number of coefficients for a tag profile in an acquired image is its $k$-space acquisition protocol. For example, the acquisition of a small region in $k$ space might skip the higher frequency harmonic peaks that are present due to the tag pattern design.

\section{METHODS}

The coefficients of the tag profiles are determined by their Fourier series expansion; therefore, it is relatively simple to determine the coefficients for a desired tag profile. For example, a specific tag profile can be designed, then the first coefficients of its Fourier series can be used as the coefficients for the harmonic images. However, implementation of these coefficients in an imaging sequence might be impossible due to limitations in the tagging sequence and restrictions on imaging time. This gap between achievable and desired tag profiles can be filled by exploiting the harmonic decomposition of the original tagged image. We now describe an approach using observed harmonic images to synthesize more detailed tag profiles.

We assume that all harmonic images $\mathbf{I}, \ell=0, \pm 1, \pm 2, \ldots$, are extracted from the observed image. Let the desired tag profile have the Fourier series coefficients $\left\{\hat{c}_{\ell}\right\}$ and $\left\{\hat{\theta}_{\ell}\right\}$, for $\ell=$ $0, \pm 0, \pm 1, \ldots, \pm \hat{L}$ where the number $\hat{L}$ could be larger than $L$. The resulting image can then be synthesized from the harmonic components of the original image by

$$
\hat{\mathbf{I}}=\sum_{\ell=0}^{L} \frac{\hat{c}_{\ell}}{c_{\ell}} \mathbf{D}_{\ell} \cos \left(\boldsymbol{\Phi}_{\ell}-\theta_{\ell}+\hat{\theta}_{\ell}\right) .
$$

In (5), the summation of the complex harmonics in (3) was replaced with the summation of real sinusoids, using the fact that the pairs of harmonic images can be simplified to a real sinusoid. This simplification provides a computational advantage as a fewer number of summations is required, hence, the change in the summation limits. Notice also in (6) the normalization of the harmonic magnitude images by dividing by $\left\{c_{\ell}\right\}$.

Since some of the original coefficients $c_{\ell}$ might be zero (as for missing harmonics in the original image) or insignificant for computations, they can be replaced by the components of the 


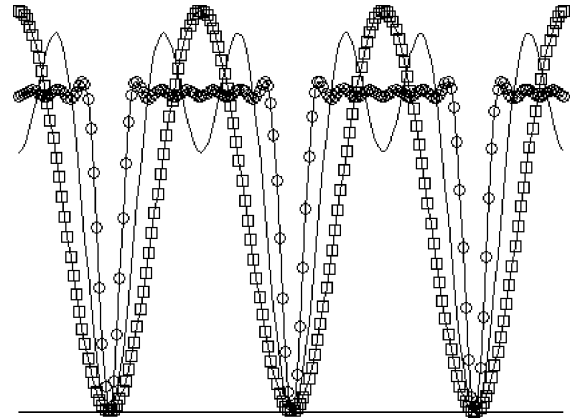

Fig. 2. Expected profiles of a tag line for different numbers of coefficients: squares for two coefficient, solid plot for four coefficients, and circles for seven coefficients.

first harmonic, which we assume to be always nonzero. Equation (6) can then be replaced by

$$
\begin{aligned}
\hat{\mathbf{I}}= & \sum_{\ell=0}^{L} \frac{\hat{c}_{\ell}}{c_{\ell}} \mathbf{D}_{\ell} \cos \left(\boldsymbol{\Phi}_{\ell}-\theta_{\ell}+\hat{\theta}_{\ell}\right) \\
& +\sum_{\ell=L}^{\hat{L}} \frac{\hat{c}}{c_{1}} \mathbf{D}_{1} \cos \left(\ell\left(\boldsymbol{\Phi}_{1}-\theta_{1}\right)+\hat{\theta}_{\ell}\right) .
\end{aligned}
$$

In the extreme case of acquiring or extracting only the first harmonic, as would be the case if 1-1 SPAMM were used, we can synthesize a more detailed tagged image from a single harmonic peak using

$$
\hat{\mathbf{I}}=\sum_{\ell=0}^{\hat{L}} \hat{c}_{\ell} \mathbf{D}_{1} \cos \left(\ell \boldsymbol{\Phi}_{1}+\hat{\theta}_{\ell}\right)
$$

where the coefficient for the original image was dropped for simplicity. A problem with this equation is that the initial coefficients need to be known, especially the phase coefficients, which are hard to obtain with good accuracy.

\section{A. Desired Tag Profile}

For the experiments detailed in this paper, a set of coefficients was required such that the desired tag profile would be sharp. The corresponding coefficients were computed from a subset of the Fourier series of a desired tag profile. The sharpness of the tag profile depends on the value and number of tag coefficients used. In Fig. 2, a set of seven coefficients with $\{3.64,1.00$, $-0.89,0.72,-0.52,0.32,-0.15\}$, normalized to the second coefficient, was used to demonstrate the effect of number of coefficients. The three plots are for the usage of two, three, and seven coefficients. As can be seen, the larger number of coefficients make the dips, corresponding to the tag lines in the resulting images, narrower. Using all seven coefficients, the resulting synthetic tagged images will have 13 peaks. Because some of the farthest harmonic peaks in the resulting image might have been outside the acquired Fourier space of the original images, the images then need to be interpolated using zero filling of the Fourier space so that the resulting harmonic images can have higher resolution.

\section{B. Tag Orientation}

The image that would be generated by (6)-(8) would contain dark lines with a specific intensity profile around these lines. The orientation of the lines, on the other hand, is determined by the location of the original harmonic peak. For example, harmonic peaks on the horizontal axis (readout direction) result in vertical tag lines.

Using the concept of harmonic peaks, we can, however, generate a new set of tag orientations different than the original ones. Assuming the two harmonic phases $\boldsymbol{\Phi}_{\ell}^{\langle v\rangle}$ and $\boldsymbol{\Phi}_{\ell}^{\langle h\rangle}$ correspond to vertical and horizontal tag lines, respectively, it is possible then to generate a harmonic phase corresponding to a diagonal tag line $\boldsymbol{\Phi}_{\ell}^{\langle/\rangle}$by adding the vertical and horizontal harmonic phases. Mathematically, we can design a new harmonic phase using (see the Appendix)

$$
\boldsymbol{\Phi}_{\ell}^{\langle/\rangle}=\Phi_{\ell}^{\langle v\rangle}+\boldsymbol{\Phi}_{\ell}^{\langle h\rangle}
$$

The other diagonal orientation can be obtained by replacing the summation operation with subtraction. In general, we can write multiple tag orientations and spacing by various combinations of the vertical and horizontal harmonic phases.

A tag grid can be generated from two images with synthetic tag lines by simple multiplication of these images (not the harmonic phase). In the results shown below, the grid images are generated by this simple operation.

\section{RESULTS AND DisCUSSION}

To demonstrate the ability of the technique to generate a synthetic crisp tag profile, different tagged MR images were used. We synthesized tagged images from three types of images: SPAMM tags, CSPAMM images, and single harmonic imaging used for Fast-HARP [16], [17].

\section{A. SPAMM Tagged Images}

These images are of a canine heart with abnormal motion and were presented before by McVeigh et al. in [18]. The initial coefficients for the original tag profile were determined from the spectral peaks in the Fourier space. Using (6), we redesigned the tag profile and the results are shown in Fig. 3. Panels (a) and (b) show the evolution tag profile as more coefficients are incorporated in tag generation. The far left images are the original images, while the far right images have the largest number of coefficients. Notice that the lines tag lines get thinner as we increase the number of coefficients.

The number of harmonic peaks in the spectrum of the synthetic tagged images is larger than that of the original image. Fig. 4(a) and (b) shows the spectrum of the vertical tagged image for the original image with zero padding and that of the synthetic image, respectively. Notice the increase in the number of harmonic peaks in the new synthetic tagged image, as expected.

\section{B. CSPAMM Image}

A CSPAMM image for a normal heart is shown in Fig. 5(a). CPAMM images have only two peaks in the Fourier space, hence the tag profile is sinusoidal. We used this data set to demonstrate the generation of a sharp tag profile, as well 

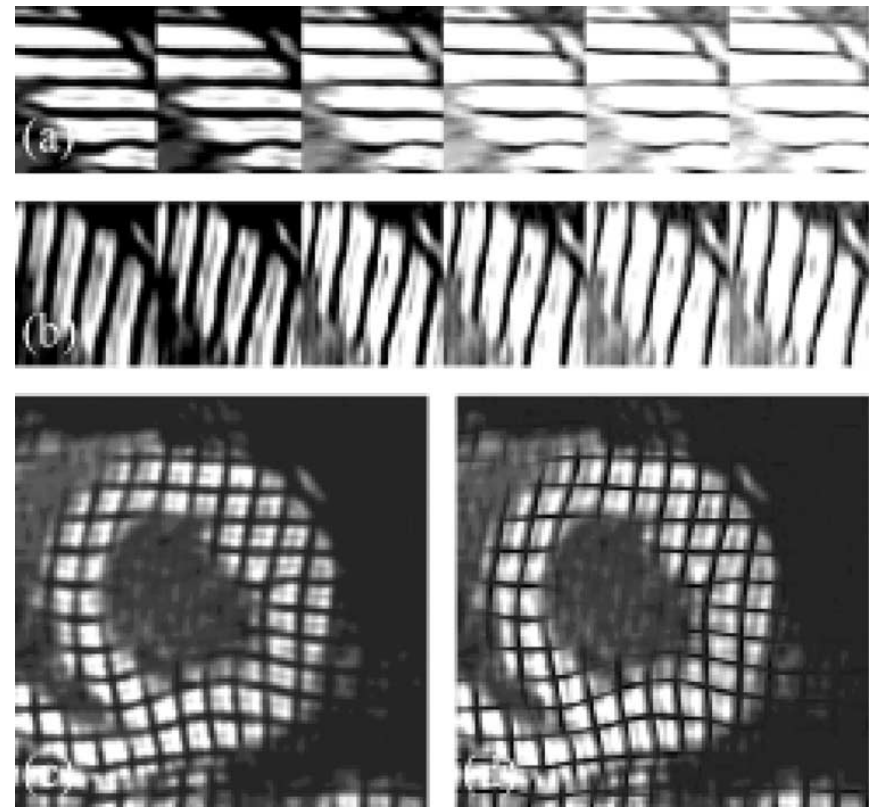

Fig. 3. (a) and (b) show the evolution tag profile with the number of coefficients. Original tagged image, composed into a grid by multiplication, is shown in (c), while the regenerated tag pattern is shown in (d).
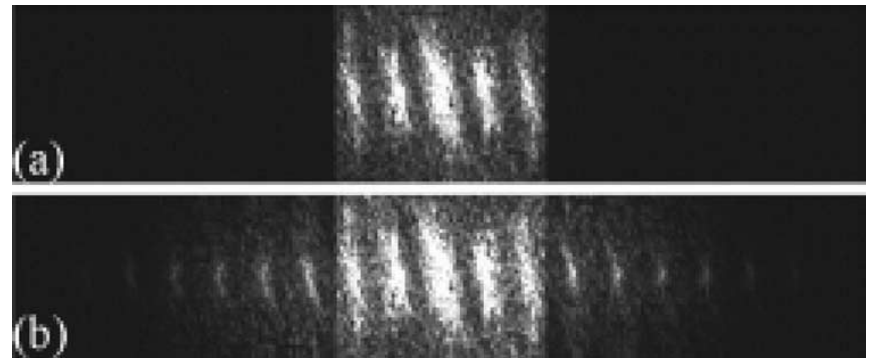

Fig. 4. (a) Spectrum of the original tagged image with vertical tag lines (b) Spectrum of the image with generated tag lines.
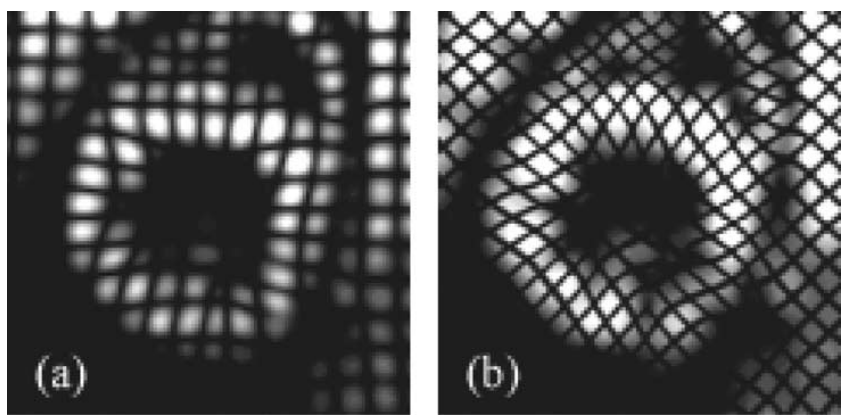

Fig. 5. (a) Original CSPAMM image, composed as a grid by multiplication (b) Regenerated tag image with diagonal tag lines and composed into a grid.

as the ability to change the orientation of the tag patterns. Diagonal HARP images are generated using (9). Then, using the formula in (8), we generated the synthetic tag images whose multiplication produced the diagonal grid shown in Fig. 5(b).

\section{Fast-HARP}

Because of the significance of a single harmonic peak in measuring motion, a special pulse sequence was designed to rapidly acquire only a single harmonic peak [16], [17]. The fast-HARP
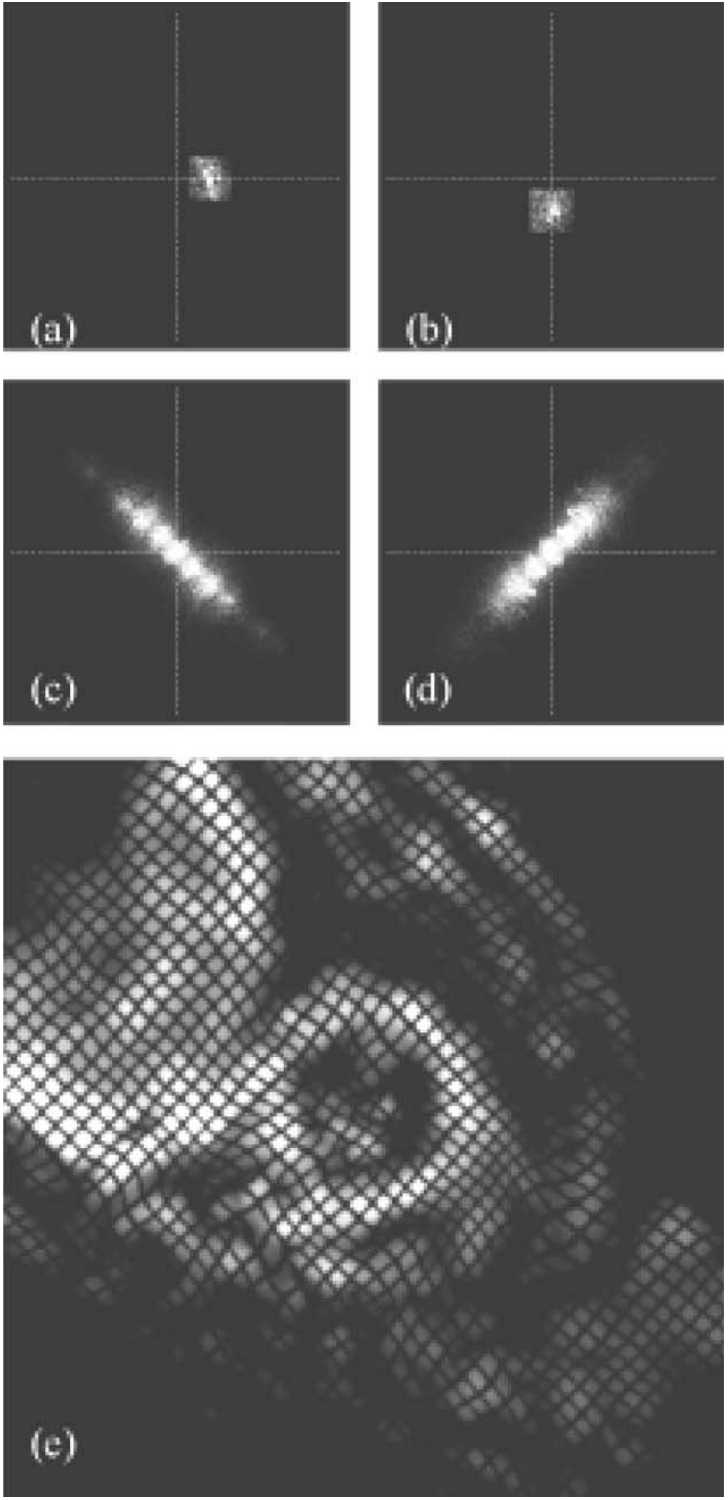

Fig. 6. (a), (b) Two peaks acquired using the fast-HARP pulse sequence for the vertical and horizontal tag orientation, respectively. (c), (d) Spectra of the resulting two synthetic tagged images with diagonal orientations. (e) Resulting grid tags.

pulse sequence is a new imaging technique for quantification of regional function of the heart in real time. The sequence acquires only a single harmonic peak several times in a single heartbeat using a modified echo planar imaging (EPI) sequence. In two heart beats, two complete sequences corresponding to vertical and horizontal tagging are acquired. This sequence is capable of acquiring a single harmonic peak in $44 \mathrm{~ms}$, thus enabling real-time imaging of the heart. The resulting harmonic image, however, is a blurred image that is difficult to visualize. Although the harmonic phase information is useful for quantifying motion, the inability to produce tagging types of images makes it hard to visualize motion.

Fig. 6(a) and (b) shows the two acquired harmonic peaks shifted from the origin in a zero padded image. Synthetic diagonally tagged images were generated using (8), and their spectra are shown in Fig. 6(c) and (d). The final synthetic image, shown in Fig. 6(e), shows blurred morphology of the heart, due to the 
reduced filter size, with a crisp diagonal tag grid. Notice that the initially acquired two peaks were used to generate all the harmonic peaks in the new synthetic tagged images. So, if they do not show up in their original form, they implicitly exist in all the harmonic peaks. This is exactly the advantage of this technique, which uses the harmonic peaks; whatever the initial generation method is, they can be used as row material to generate a wide variety of tag patterns.

It is important to mention that in this paper we considered that the harmonic images correspond to the harmonic peaks simply by filtering. However, the real spectrum of a harmonic peak is not band limited and is spread out to infinity. This means that the images produced by filtering are corrupted by the filtering process, as it cuts some part of the spectrum, and by contamination by signal from the other harmonic peaks (which we call interference). The extent of the corruption depends on how close the peaks are to each other; the closer they are, the more corrupted the resulting images are. Luckily, the distance between the peaks can be controlled by changing the tag separation, and a tag separation of $8 \mathrm{~mm}$ is basically sufficient to limit the extent of artifacts.

Other sources of corruption that were not mentioned in the paper, for the sake of simplicity, are the effects of coil sensitivity and field inhomogeneity. These factors are especially effective in the case of using Fast-HARP data. The effect of these artifacts needs to be studied in the future in order to reduce any resulting artifacts.

\section{CONCLUSION}

In this paper, we propose a technique to regenerate a large number of tag patterns from an original tagged image with simple line tags. The technique is based on HARP concepts that have been demonstrated to be useful for measuring motion. The tag synthesis was demonstrated on examples of tagged images. The technique is useful for refining the tag pattern of other motion analysis techniques that identify tag lines from the intensity image directly. Another application is for image compression. In this case, only the basic harmonic components can be used to generate a large number of tag patterns. The tag synthesis method can be used in applications that collect data using stimulated echo and methods like DENSE. The acquired images are also complex images similar to the harmonic components.

\section{APPENDIX}

The relation between the harmonic phase and the motion of the tags can be described mathematically by (cf., [6], [7], and [13])

$$
\boldsymbol{\Phi}_{\ell}^{\langle v\rangle}(\mathbf{y}, t)=\mathbf{q}(\mathbf{y}, t)^{T} \boldsymbol{\omega}_{\ell}^{\langle v\rangle}
$$

where $\mathbf{q}(\mathbf{y}, t)$ is a function that describes the 2-D motion of the points $\mathbf{y}$, on the image plane at time $t$, and $\omega_{\ell}^{\langle v\rangle}$ is a vector that describes the location of the harmonic peak. A similar equation can be obtained for the HARP image, $\boldsymbol{\Phi}_{\ell}^{\langle h\rangle}$, with the horizontal vector, $\omega_{\ell}^{\langle h\rangle}$. The diagonal harmonic phase then is

$$
\boldsymbol{\Phi}_{\ell}^{\langle/\rangle}=\boldsymbol{\Phi}_{\ell}^{\langle v\rangle}+\boldsymbol{\Phi}_{\ell}^{\langle h\rangle}=\mathbf{q}^{T}\left(\boldsymbol{\omega}_{\ell}^{\langle v\rangle}+\boldsymbol{\omega}_{\ell}^{\langle h\rangle}\right)=\mathbf{q}^{T} \boldsymbol{\omega}_{\ell}^{\langle/\rangle}
$$

which demonstrates that it describes the motion component in the diagonal direction.

In general, we can generate images using the combination

$$
m \boldsymbol{\Phi}_{\ell}^{\langle v\rangle}+n \boldsymbol{\Phi}_{\ell}^{\langle h\rangle}=\mathbf{q}^{T}\left(m \boldsymbol{\omega}_{\ell}^{\langle v\rangle}+n \boldsymbol{\omega}_{\ell}^{\langle h\rangle}\right)
$$

where $m$ and $n$ are integers. The corresponding harmonic peaks for the $(m, n)$ combinations are on a regular grid spanned by the original two vectors $\boldsymbol{\omega}_{\ell}^{\langle v\rangle}$ and $\boldsymbol{\omega}_{\ell}^{\langle h\rangle}$.

\section{ACKNOWLEDGMENT}

The authors would like to thank Dr. E. McVeigh for providing the dog images, Dr. D. Kraitchman, S. Sampath, and Dr. J. A. Derbyshire (NHLBI) for providing the fast-HARP images, and S. Ryf (Institute for Biomedical Engineering, University and ETH Zurich, Switzerland) for providing the CSPAMM images.

\section{REFERENCES}

[1] L. Axel and L. Dougherty, "MR imaging of motion with spatial modulation of magnetization," Radiology, vol. 171, pp. 841-5, 1989.

[2] - "Heart wall motion: Improved method of spatial modulation of magnetization for MR imaging," Radiology, vol. 172, pp. 349-50, 1989.

[3] E. A. Zerhouni, D. M. Parish, W. J. Rogers, A. Yang, and E. P. Shapiro, "Human heart: Tagging with MR imaging - A method for noninvasive assessment of myocardial motion," Radiology, vol. 169, pp. 59-63, 1988.

[4] L. Axel, R. C. Goncalves, and D. Bloomgarden, "Regional heart wall motion: Two-dimensional analysis and functional imaging with MR imaging," Radiology, vol. 183, pp. 745-50, 1992.

[5] T. S. Denney Jr, "Estimation and detection of myocardial tags in MR image without user-defined myocardial contours," IEEE Trans. Med. Imag., vol. 18, pp. 330-44, Apr. 1999.

[6] N. F. Osman, W. S. Kerwin, E. R. McVeigh, and J. L. Prince, "Cardiac motion tracking using CINE harmonic phase (HARP) magnetic resonance imaging," Magn. Reson. Med., vol. 42, pp. 1048-60, 1999.

[7] N. F. Osman, E. R. McVeigh, and J. L. Prince, "Imaging heart motion using harmonic phase MRI," IEEE Trans. Med. Imag., vol. 19, pp. 186-202, Mar. 2000.

[8] J. L. Prince, S. N. Gupta, and N. F. Osman, "Bandpass optical flow for tagged MRI," Med. Phys., vol. 27, pp. 108-18, 2000.

[9] Y. P. Wang, Y. Chen, and A. A. Amini, "Fast LV motion estimation using subspace approximation techniques," IEEE Trans. Med. Imag., vol. 20, pp. 499-513, June 2001.

[10] M. Stuber, M. A. Spiegel, S. E. Fischer, M. B. Scheidegger, P. G. Danias, E. M. Pedersen, and P. Boesiger, "Single breath-hold slice-following CSPAMM myocardial tagging," Magma, vol. 9, pp. 85-91, 1999.

[11] S. E. Fischer, G. C. McKinnon, S. E. Maier, and P. Boesiger, "Improved myocardial tagging contrast," Magn. Reson. Med., vol. 30, pp. 191-200, 1993.

[12] J. P. Kuijer, E. Jansen, J. T. Marcus, A. C. van Rossum, and R. M. Heethaar, "Improved harmonic phase myocardial strain maps," Magn. Reson. Med., vol. 46, pp. 993-9, 2001.

[13] N. F. Osman and J. L. Prince, "Visualizing myocardial function using HARP MRI," Phys. Med. Biol., vol. 45, pp. 1665-82, 2000.

[14] T. J. Mosher and M. B. Smith, "A DANTE tagging sequence for the evaluation of translational sample motion," Magn. Reson. Med., vol. 15, pp. 334-9, 1990.

[15] W. S. Kerwin and J. L. Prince, "A $k$-space analysis of MR tagging," $J$ Magn. Reson., vol. 142, pp. 313-22, 2000.

[16] D. L. Kraitchman, S. Sampath, E. Castillo, J. A. Derbyshire, R. C. Boston, D. A. Bluemke, B. L. Gerber, J. L. Prince, and N. F. Osman, "Quantitative ischemia detection during cardiac magnetic resonance stress testing by use of fastHARP," Circulation, vol. 107, pp. 2025-2030, 2003. 
[17] S. Sampath, J. Derbyshire, N. Osman, E. Atalar, and J. Prince, "Real-time imaging of cardiac strain using ultra fastHARP sequence," in Proc. ISMRM 9th Scientific Meeting Exhibition 2001, Glasgow, U.K., 2001.

[18] E. R. McVeigh, F. W. Prinzen, B. T. Wyman, J. E. Tsitlik, H. R. Halperin, and W. C. Hunter, "Imaging asynchronous mechanical activation of the paced heart with tagged MRI," Magn. Reson. Med., vol. 39, pp. 507-13, 1998.

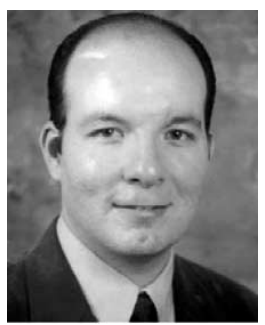

Nael F. Osman received the B.Sc. and M.S. degrees from the Department of Electronics and Communications, Faculty of Engineering, Cairo University, Egypt, in 1992 and 1995, respectively. He receive the Ph.D. degree in electrical and computer engineering from Johns Hopkins University, Baltimore, MD.

Currently, he is an Assistant Professor in the Department of Radiology and Radiological Science, Johns Hopkins University. He also holds a joint appointment in the Department of Electrical and Computer Engineering. His research interests include signal and image processing, medical imaging, and magnetic resonance imaging (MRI). His special interests are in noninvasive imaging of mechanical properties of tissue and quantitative assessment of regional functions of the heart.

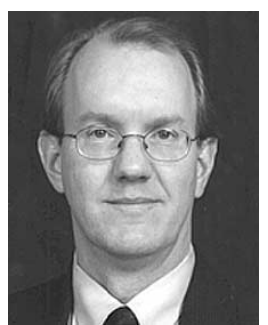

Jerry L. Prince (S'78-M'83-SM'96-F'04) received the B.S. degree from the University of Connecticut in 1979 and the S.M., E.E., and Ph.D. degrees in 1982, 1986, and 1988, respectively, from the Massachusetts Institute of Technology, Cambridge, all in electrical engineering and computer science.

He joined the technical staff at The Analytic Sciences Corporation (TASC) in Reading, MA, where he contributed to the design of an automated vision system for synthetic aperture radar imaging. He joined the faculty at the Johns Hopkins University, Baltimore, MD, in 1989, and has been a Professor in the Department of Electrical and Computer Engineering since 1998. He holds joint appointments in the Department of Radiology, the Department of Biomedical Engineering, and the Department of Applied Mathematics and Statistics. Since 2001, he has been the Associate Director for Research of the Engineering Research Center for Computer Integrated Surgical Systems and Technology. Over the past 15 years, he has published numerous book chapters and peer-reviewed journal and conference papers. He co-edited Measurement of Cardiac Deformations from MRI: Physical and Mathematical Models (with A. A. Amini) (Dordrecht, The Netherlands: Kluwer). His current research interests include image processing and computer vision with application to medical imaging. Major projects include magnetic resonance imaging of cardiac motion, three-dimensional brain image analysis, vector tomography, and computer integrated surgery.

Dr. Prince has served as Associate Editor for IEEE TRANSACTIONS ON IMAGE PROCESSING and is currently an Associate Editor for IEEE TRANSACTIONS ON MEDICAL IMAGING. He is also on the Editorial Board of Medical Image Analysis. $\mathrm{He}$ is a member of Sigma Xi professional society and Tau Beta Pi, Eta Kappa Nu, and Phi Kappa Phi honor societies. He was a member of the IEEE Signal Processing Society Image and Multidimensional Signal Processing (IMDSP) Technical Committee, from 1994 to 2000. He has received several awards, including the 1993 National Science Foundation Presidential Faculty Fellows Award and the 1997 Maryland Outstanding Young Engineer Award. 